Physica A 160 (1989) 471-481

North-Holland, Amsterdam

\title{
A GENERALIZED VLASOV EQUATION
}

\author{
Ademir E. SANTANA, A. MATOS NETO and J.D.M. VIANNA ${ }^{1}$ \\ Universidade Federal da Bahia, Instituto de Física, Campus de Ondina, 40210, Salvador, BA, \\ Brazil
}

Received 1 April 1989

\begin{abstract}
A two-spacial dimension electronic system characterized by a plasma parameter $\Gamma \leqslant 1$ is analyzed; then, by using a rigorous non-equilibrium statistical mechanical theory, the evolution of distribution function is considered. A generalized Vlasov equation (GVE) is derived. Compared to the usual Vlasov equation, GVE presents an additional velocitydependent correlation term. Taking as a starting point the GVE, the phenomenological approximation to two-particles function, $f_{2}\left(r_{1} r_{2} p_{1} p_{2} ; t\right)=f_{1}\left(r_{1} p_{1} ; t\right) f_{1}\left(r_{2} p_{2} ; t\right) g\left(r_{1}-r_{2}\right)$, proposed by Singwi, Tosi, Landi and Sjolander is analyzed.
\end{abstract}

\section{Introduction}

Properties of low-dimensional electronic systems have been extensivcly studied in the literature [1-5]. Particularly important in this area is the system formed by electrons on a helium surface. Such a system we will call in this paper TDES (two-dimensional electronic system).

TDES is a system with correlational properties; it is characterized by an electron density $c$ in the experimental range $10^{5} \leqslant c \leqslant 10^{9}[4,5]$. In this situation the range of electrostatic energy per particle $\left(2 \times 10^{-16}\right.$ ergs to $7 \times 10^{-15}$ ergs $)$ is comparable to a typical thermal energy $\left(\sim 1.4 \times 10^{-16}\right.$ ergs $)$. Then, the quantum effects are not important and TDES exhibits a classical behaviour, i.e. TDES can be treated as a classical plasma. Indeed, the electron system behaves like a non-degenerate plasma described hy a two-dimensional Boltzmann distribution as a limit of the Fermi distribution at experimental densities and temperatures.

From a theoretical standnoint, the TDES has been studied by several authors [6-11]. In particular, Studart and Hipólito [12,13] have considered the correlation effects by using the self-consistent field approximation proposed by Singwi, Tosi, Land and Sjolander (STLS) [14]. In the STLS approximation, the

\footnotetext{
${ }^{1}$ Permanent adciress. Departamento de Física, Instituto de Ciencias Exatas, Universidade de Brasília, 70910, Brasilia, DF, Brazil.
} 
two-particle distribution function $f_{2}\left(r_{1}, r_{2}, v_{1}, v_{2} ; t\right)$ is written as

$$
f_{2}\left(r_{1}, r_{2}, v_{1}, v_{2} ; t\right)=f_{1}\left(r_{1}, v_{1} ; t\right) f_{1}\left(r_{2}, v_{2} ; t\right) g\left(\left|r_{1}-r_{2}\right|\right),
$$

where $f_{1}(r, v ; t)$ is the single-particle distribution function and $g\left(\left|r_{1}-r_{2}\right|\right)$ is the static pair correlation function.

The STLS method is strongly based on the relation (1) which is a phenomenological hypothesis. Indeed, in this scheme the equation of motion of the one-particle distribution function is truncated under the assumption that the coupling of the particle to the medium is given by the function $g(r)$.

It is known that the main object of a partly intuitive or semi-phenomenological model is the calculation of the physical quantities from its fundamental equations, which are accepted more or less a priori. In consequence, one has no: an analytical method to modify the equations of such models in order to obtain the best results for a given problem. The STLS model as it is presented in the literature can be included in this category of phenomenological models.

In the present paper, by using a rigcrous non-equilibrium statistical mechanical theory, we will analyze relation (1). Our starting point is the dynamics of correlations (DC) developed mainly by Prigogine and Balescu $[15,16]$. The DC has, at least, two aspects which are interesting to the study of the STLS approximation: (i) the DC has been used with success in the study of plasma $[16,17]$ (ii) by using DC theory one obtains the same diagrammatical representation for both classical and quantum plasmas [16]. This aspect (ii) can be useful in the analysis of the classical limit of a quantum system.

We will consider here the TDES characterized by a plasma parameter $\Gamma \approx 1$. The parameter $\Gamma$ is defined as $\Gamma=\langle V\rangle /\langle K\rangle$ where $\langle V\rangle$ and $\langle K\rangle$ ainote the averages of the potential and kinetic energies respectively. We will show that the correlation effects will be related to diagrams we will call 2 nd order diagrams. Our analysis concerns to derivation of an equation which describes the behavior of the plasma over short periods of time, i.e., for $t=\mathscr{O}\left(t_{\mathrm{p}}\right)$, where $t_{\mathrm{p}}$ is the plasma oscillation period, but since we will consider the case in which $\Gamma$ is no longer very small we will need to include terms of order $e^{2}\left(e^{2} c\right)^{n}$. As a result, we obtain a generalized Vlasov equation (GVE). Compared to the usual Vlasov equation our equation presents an additional velocity-dependent correlation term. Taking as a starting point the GVE we will obtain, as a particular case, the equation related to hypothesis (1). Hence, we can show the principles guiding the choice of the diagrams in the STLS model; this feature allows to us to indicate as the STLS model can be improved.

In section 2 we present the notation we have used in the paper, the characteristic properties of TDES and the diagrams necessary to describe TDES by using DC theory. In section 3 we will derive the GVE. Section 4 
contains an analysis of the STLS approximation seen as a particular case of our GVE. In section 5 we present final remarks and conclusions.

\section{Notation and preliminaries}

The TDES will be consiaered here as a two-dimensional $N$-particle system with interaction potential given by

$$
V_{i j}=e /\left|r_{i}-r_{j}\right|, \quad r=\hat{\imath} x+\hat{\jmath} y, \quad i, j=1, \ldots, N,
$$

where $e=e_{0}[2 /(1+\varepsilon)]^{1 / 2}$ with $e_{0}$ the electronic charge and $\varepsilon$ the dielectric function. The system occupies a volume $\Omega$. The perturbative solution of the Liouville equation in Fourier representation is, according to DC theory [16], given by

$$
\rho_{\{k\}}(v ; t)=-\frac{1}{2 \pi \mathrm{i}} \sum_{\left\{k^{\prime}\right\rangle} \sum_{n=0}^{\infty}\left(-e^{n}\right)\left\langle\{k\}\left|R_{0}(\omega)\left[L^{\prime} R_{0}(\omega)\right]^{n}\right|\left\{k^{\prime}\right\}\right\rangle \rho_{\left\{k^{\prime}\right\}}(v ; 0) .
$$

The coefficients $\rho_{\{k\}}$ are factored as [16]

$$
\rho_{k_{1}, k_{2}, \ldots, k_{s}}(1, \ldots, s)=\prod_{j=1}^{s} \rho_{k_{j}}(j)+\rho_{\left[k_{1} \ldots k_{s}\right]}(1, \ldots, s) .
$$

The first term in eq. (4) is a product of $s$ non-homogeneous factors. The second is the sum of all possible correlation patterns of $s$ particles.

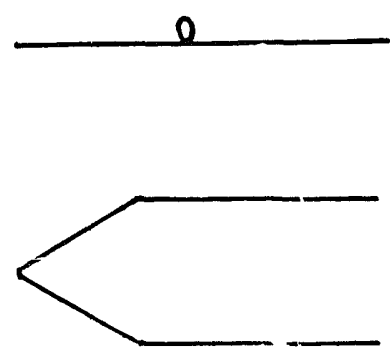

A

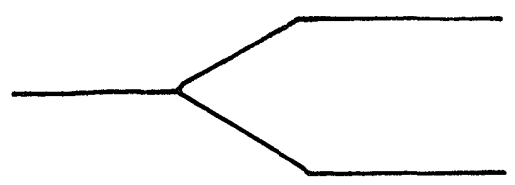

C

Fig. 1. Vertices which compose the relevant diagrams for calculation of $\rho_{11}$ and $\rho_{k}$. 
Associated with eq. (3) there is a set of diagrams $[15,16]$. The choice of relevant diagrams must be consistent with specifications of the studied system. In our case, the TDES is characterized by $\Gamma \leqslant 1$, and we will be interested here in a short-time process. Thus, the main contributions at eq. (3) will be diagrams corresponding to order $\left(e^{2} c\right)^{n}$, which we will call first order diagrams, and $e^{2}\left(e^{2} c\right)^{n}$, which we will call second order diagrams, being $c=N / \delta 2$. The vertices of these diagrams are presented in fig. 1 . Once the relevant diagrams have been chosen, we can make their summation in order to determine their contribution to $\rho_{0}(t)$ and $\rho_{k}(t)$.

\section{A generalized Vlasov equation}

\subsection{First and second order contributions to $\rho_{0}$}

The contributions to $\rho_{0}$ are derived from diagrms that have not external lines at left, i.e. the relevant diagrams are composed by $A, B$ and $C$ vertices shown in fig. 1. The vertices $A$ and $C$ are connected to $B$ as is indicated in fig. 2.

Considering the summation of all the diagrams of fig. 2 the $B$ vertices at the left can be factorized and we obtain for $\rho_{0}(t)$ the result

$$
\begin{aligned}
\rho_{0}(\mid \ldots ; t)= & -\frac{1}{2 \pi} \mathrm{i}\left(-e^{2}\right) \int \mathrm{d} \omega \mathrm{e}^{\mathrm{i} \omega t} \sum_{k_{n}^{\prime}} \sum_{n, j} F_{j n}\left(-k_{n}^{\prime}\right)\left(\boldsymbol{k}_{n}^{\prime} \cdot \boldsymbol{v}_{n}-\boldsymbol{k}_{n}^{\prime} \cdot \boldsymbol{v}_{j}-\omega\right)^{-1} \\
& \times\left\{\rho_{k_{n}^{\prime}-\boldsymbol{k}_{n}^{\prime}}(n, j \mid \ldots ; 0)\right. \\
& +\left(-e^{2}\right)^{2} \sum_{a, b} F_{j a}\left(-k_{n}^{\prime}\right)\left(k_{n}^{\prime} \cdot \boldsymbol{v}_{n}-\boldsymbol{k}_{n}^{\prime} \cdot \boldsymbol{v}_{a}-\omega\right)^{-1} \\
& \times F_{a b}\left(-\boldsymbol{k}_{n}^{\prime}\right)\left(\boldsymbol{k}_{n}^{\prime} \cdot \boldsymbol{v}_{n}-\boldsymbol{k}_{n}^{\prime} \cdot \boldsymbol{v}_{b}-\omega\right)^{-1} \rho_{k_{n}^{\prime}, \boldsymbol{k}_{n}^{\prime}}(b, n \mid \ldots ; 0) \\
& \left.+\left(-e^{2}\right)^{3} \sum_{a, b, c} \ldots\right\},
\end{aligned}
$$

where

$$
F_{j n}\left(\boldsymbol{k}_{j}\right)=-\frac{4 \pi^{2}}{\Omega} V_{k j}\left(m^{-1} \boldsymbol{k}_{j} \cdot \boldsymbol{\partial}_{j n}\right)
$$

and we have used the notation of ref. [16].

We now differentiate Eq. (5) with respect to time and integrate over all velocities except $\boldsymbol{v}_{\alpha}$. The result is 

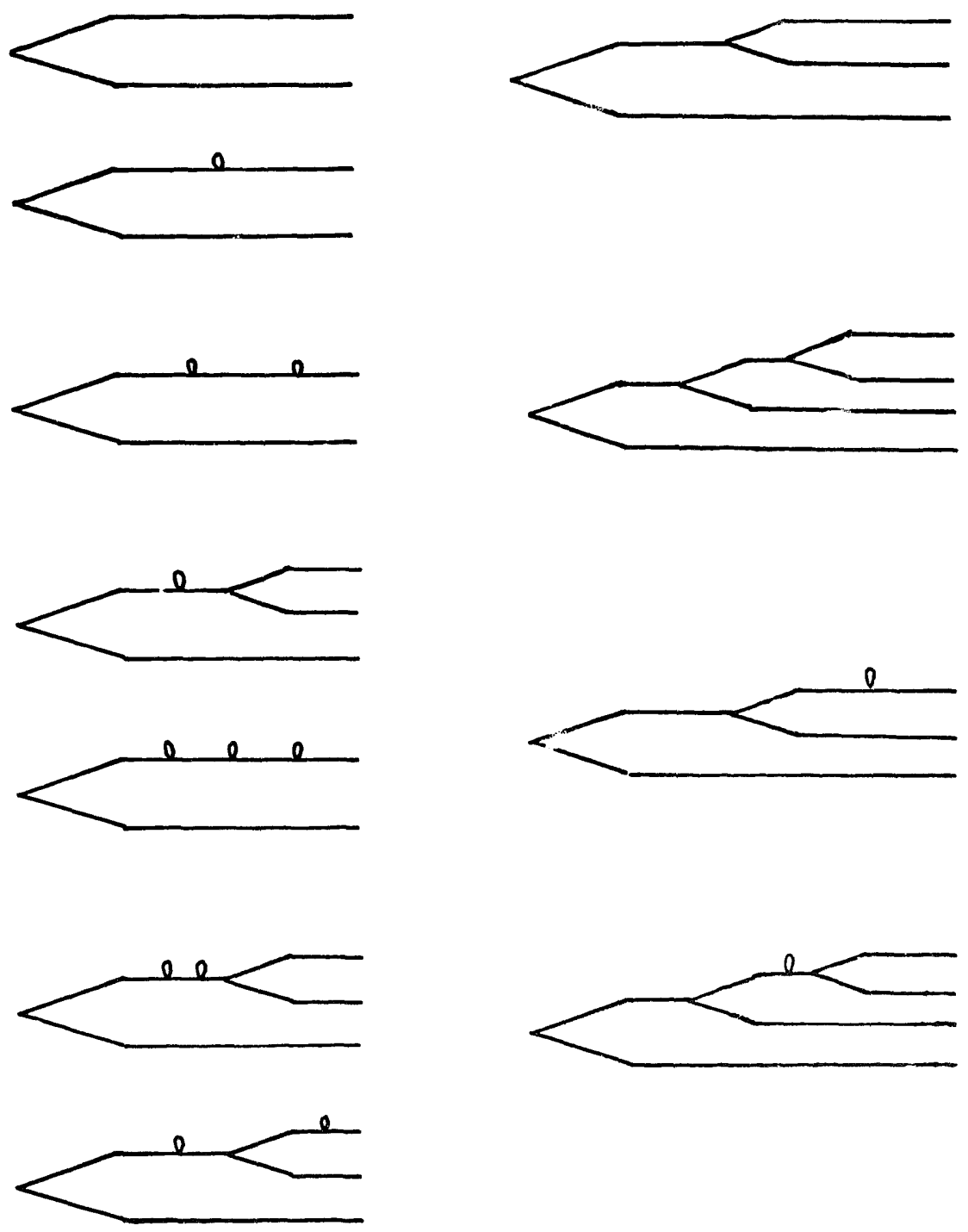

Fig. 2. Diagrams which give the relevant first and second order contributions to $\rho_{9}$.

$\partial_{t} \rho_{0}\left(\mid v_{\alpha} ; t\right)=0$

i.e., to this order of approximation, $\rho_{0}$ remains constant in time.

\subsection{First and second order contributions to $\rho_{k}$}

The contributions to $\rho_{k}$ are derived from diagrams ending at the left with one line. Then, the relevant diagrams are composed by $A$ and $C$ vertices. A general diagram in this case is represented in fig. 3 and its corresponding mathematical expression is 


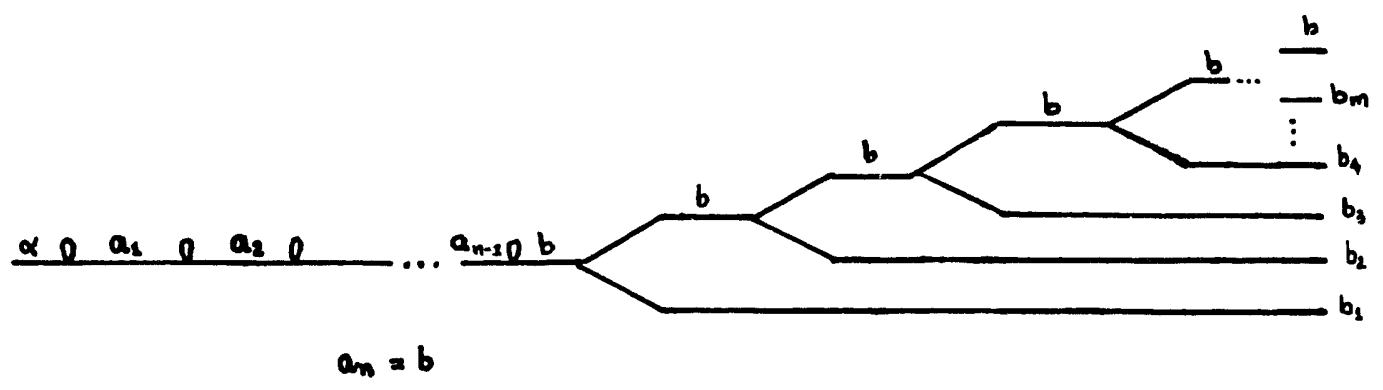

Fig. 3. A general diagram for first and second order contributions to $\rho_{k}$.

$$
\begin{aligned}
\rho_{k_{\alpha}}^{(g)}= & \frac{-\left(-e^{2}\right)^{n+m}}{2 \pi \mathrm{i}} \int \mathrm{d} \omega \mathrm{e}^{-\mathrm{i} \omega t} \\
& \times \sum_{a_{1}, \ldots, a_{n-1}, b} \sum_{b_{1}, \ldots b_{m}} \sum_{k_{b_{1}}, \ldots, k_{b_{m}}}\left(k_{\alpha} \cdot v_{\alpha}-\omega\right)^{-1} \\
& \times F_{\alpha, a_{1}}\left(k_{\alpha}\right)\left(k_{\alpha} \cdot v_{a_{1}}-\omega\right)^{-1} F_{a_{1} a_{2}}\left(k_{\alpha}\right) \cdots \\
& \cdots F_{a_{n-1}, b}\left(k_{\alpha}\right)\left(k_{\alpha} \cdot v_{b}-\omega\right)^{-i} D_{b, b_{1}}\left(k_{\alpha}-k_{b_{1}} ; k_{\alpha}\right) \\
& \times\left[\left(k_{\alpha}-k_{b_{1}}\right) \cdot v_{b}+k_{b_{1}} \cdot v_{b_{1}}-\omega\right]^{-1} D_{b, b_{2}}\left(k_{\alpha}-k_{b_{1}}-k_{b_{2}} ; k_{\alpha}-k_{b_{1}}\right) \cdots \\
& \cdots D_{b, b_{m}}\left(k_{\alpha}-k_{b_{1}}-\cdots-k_{b_{m}} ; k_{\alpha}-k_{b_{1}}-\cdots-k_{b_{m-1}}\right) \\
& \times\left[\left(k_{\alpha}-\sum_{j=1}^{m} k_{b_{j}}\right) \cdot v_{b}+\sum_{j=1}^{m-1} k_{b_{j}} \cdot v_{b_{j}}-\omega\right]^{-1} \\
& \times \rho_{k_{\alpha}-k_{b_{1}}-\cdots-k_{b_{m}}, k_{b_{m}}, \ldots, k_{b_{1}}}\left(\boldsymbol{v}_{b}, \boldsymbol{v}_{b_{1}}, \ldots, \boldsymbol{v}_{b_{m}} \mid \ldots, 0\right),
\end{aligned}
$$

where

$$
D_{j n}\left(k_{j}^{\prime}, k_{j}\right)=-\frac{4 \pi^{2}}{\Omega} V_{\left|\boldsymbol{k}_{j}^{\prime}-\boldsymbol{k}_{j}\right|} m^{-1}\left(\boldsymbol{k}_{j}^{\prime}-\boldsymbol{k}_{j}\right) \cdot \boldsymbol{\partial}_{j n} .
$$

The other relevant diagrams are obtained inserting $\mathrm{A}$ vertices between $\mathrm{C}$ vertices in fig. 3 . Correspondingly we must introduce the mathematical expression associated with the $A$ vertex in eq. (7). As an example, in fig. 4, some general diagrams in this case are shown. IVe can add all those diagrams and we obtain $\rho_{k}$ given by

$$
\begin{aligned}
\rho_{k_{\alpha}}(\alpha \mid \ldots ; t)= & -\frac{1}{2 \pi \mathrm{i}} \int \mathrm{d} \omega \mathrm{e}^{-i \omega t}\left(k_{\alpha x} \cdot v_{\alpha}-\omega\right)^{-1} \rho_{k_{\alpha}}(\alpha \mid \ldots ; 0) \\
& +\frac{\left(-e^{2}\right)}{2 \pi \mathrm{i}} \int \mathrm{d} \omega \mathrm{e}^{-i \omega t}\left(k_{\alpha} \cdot v_{\alpha}-\omega\right)^{-1} \sum_{a_{1}} F_{\alpha 1} a_{1}\left(k_{\alpha}\right)
\end{aligned}
$$



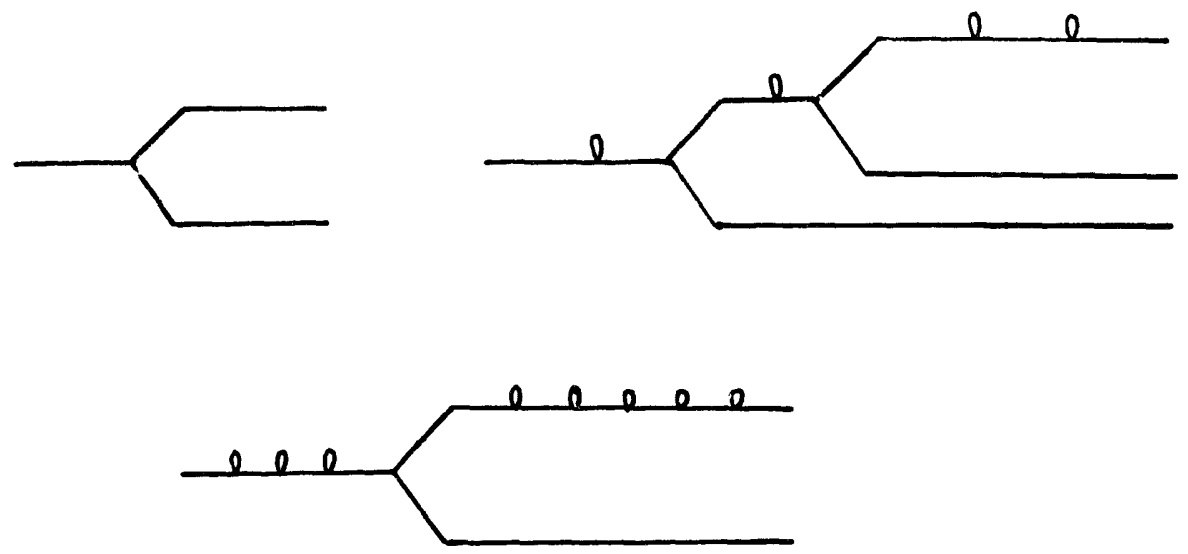

Fig. 4. Examples of diagrams obtained inserting A vertices between $\mathrm{C}$ vertices in the diagrams of fig. 3.

$$
\begin{aligned}
& \times\left\{\left(k_{\alpha} \cdot v_{a_{1}}-\omega\right)^{-1}\left[\rho_{k_{\alpha}}\left(a_{1}\right] \ldots ; 0\right)+\cdots\right. \\
& +\left(-e^{2}\right) \sum_{a_{2}} F_{a_{1} a_{2}}\left(k_{\alpha}\right)\left(k_{\alpha} \cdot v_{a_{2}}-\omega\right)^{-1} \rho_{k_{i t}}\left(a_{2} \mid \ldots ; 0\right)+\ldots \\
& +\left(-e^{2}\right) \sum_{\boldsymbol{k}^{\prime}} \sum_{a_{2}} D_{a_{1} a_{2}}\left(\boldsymbol{k}_{\alpha}, \boldsymbol{k}^{\prime}\right)\left[\boldsymbol{k}^{\prime} \cdot \boldsymbol{v}_{a_{2}}+\left(\boldsymbol{k}_{\alpha}-\boldsymbol{k}^{\prime}\right) \cdot \boldsymbol{v}_{a_{1}}-\omega\right]^{-1} \\
& \left.\left.\times \rho_{k_{\alpha}-k^{\prime}, k_{\alpha}}\left(a_{1}, a_{2} \mid \ldots ; 0\right)+\cdots\right]\right\} \\
& -\frac{\left(-e^{2}\right)}{2 \pi \mathrm{i}} \int \mathrm{d} \omega \mathrm{e}^{-\mathrm{i} \omega t}\left(\boldsymbol{k}_{\alpha} \cdot \boldsymbol{v}_{\alpha}-\omega\right)^{-1} \sum_{k^{\prime}} \sum_{a_{1}} D_{\alpha a_{1}}\left(\boldsymbol{k}_{\alpha \alpha}, \boldsymbol{k}^{\prime}\right) \\
& \times\left\{\left[\boldsymbol{k}^{\prime} \cdot \boldsymbol{v}_{\alpha}+\left(\boldsymbol{k}_{\alpha}-\boldsymbol{k}^{\prime}\right) \cdot \boldsymbol{v}_{a_{1}}\right]^{-1}\right. \\
& \left.\times \rho_{k^{\prime}, k_{\alpha}-k^{\prime}}\left(\alpha, a_{1} \mid \ldots ; 0\right)+\cdots\right\} \text {. }
\end{aligned}
$$

We can now differentiate the two sides of (8) with respect to time, integrate over all velocities except $\boldsymbol{v}_{r}$ and take the "imit $N, \Omega \rightarrow \infty ;(N / \Omega) \sim c<\infty$. As a result we obiain

$$
\begin{aligned}
& \partial_{t} \rho_{k}\left(\alpha_{i} t\right)+\mathrm{i} k \cdot v_{\alpha} \rho_{k}(\alpha ; t) \\
& \quad-4 \pi^{2} e^{2} c m^{-1}\left\{V_{k} \mathrm{i} k \cdot \partial_{\alpha} \int \mathrm{d} v_{j} \rho_{k}\left(v_{j} \mid v_{\alpha} ; t\right)\right. \\
& \left.\quad+\partial_{\alpha} \cdot \int \mathrm{d} k^{\prime} \mathrm{d} v_{j} \mathrm{i}\left(k-k^{\prime}\right) V_{\left|k-k^{\prime}\right|} \rho_{k^{\prime}}(\alpha ; t) \rho_{k-k^{\prime}}(j ; t)\right\} \\
& \quad=I_{\mathrm{cor}}\left(k, v_{\alpha} ; t\right)
\end{aligned}
$$


with

$$
\begin{aligned}
I_{\text {cor }}\left(k, v_{\alpha} ; t\right)= & 4 \pi^{2} e^{2} c m^{-1} \partial_{\alpha} \cdot \int \mathrm{d} k^{\prime} \mathrm{d} v_{j} \mathrm{i}\left(k-k^{\prime}\right) \\
& \times V_{\left|k-k^{\prime}\right|} \rho_{\left[k^{\prime}, k-k^{\prime}\right]}\left(v_{\alpha}, v_{j} ; t\right)
\end{aligned}
$$

where we have used eq. (4) to obtain eq. (9) and eq. (10).

Eq. (9) can be transformed to phase space, by multiplying both sides with $\left(\Omega / 4 \pi^{2}\right) \exp \left(\mathrm{i} k \cdot x_{\alpha}\right)$ and integrating over $k$. Hence, we obtain

$$
\begin{aligned}
& \partial_{t} f_{1}\left(v_{\alpha}, r_{\alpha} ; t\right)+v_{\alpha} \cdot \partial_{\alpha} f_{1}\left(v_{\alpha}, r_{\alpha} ; t\right) \\
& \quad-e^{2} m^{-1}\left[\partial_{\alpha} f_{1}\left(v_{\alpha}, r_{\alpha} ; t\right)\right] \cdot \nabla_{\alpha} \int \mathrm{d} r_{j} \mathrm{~d} v_{j} V\left(\left|r_{\alpha}-r_{j}\right|\right) \\
& \quad \times\left[f_{1}\left(r_{j}, v_{j} ; t\right)-c \varphi\left(v_{j} ; t\right)\right] \\
& \quad=I_{\text {cor }}\left(r_{\alpha}, v_{\alpha} ; t\right)
\end{aligned}
$$

where $\varphi(v ; t)$ is the one-particle velocity distribution function and

$$
I_{\text {cor }}\left(r_{\alpha}, v_{\alpha} ; t\right)=\frac{\Omega}{4 \pi^{2}} \int \mathrm{d} k \mathrm{e}^{\mathrm{i} k \cdot r_{\alpha}} I_{\text {cor }}\left(\tilde{k}, v_{\alpha} ; t\right) .
$$

The left-hand side in eq. (11) is the equation first proposed by Vlasov [18]. The right-hand side represents a correction to the Vlasov equation. By our development this term is originated from $\rho_{\left[k^{\prime}, k-k^{\prime}\right]}$, i.e, it is asssociated with true correlations. Eq. (11) we will call generalized Vlasov equation (GVE).

\section{Relation between the GVE and the STLS approximation}

Considering eq. (1), the first BBGKY equation [19] can be written as

$$
\begin{aligned}
& \partial_{t} f_{1}\left(r_{\alpha}, v_{\alpha} ; t\right)+v_{\alpha} \cdot \nabla_{\alpha} f_{1}\left(r_{\alpha}, v_{\alpha} ; t\right) \\
& \quad-e^{2} m^{-1} \partial_{\alpha} \cdot \int \mathrm{d} r \mathrm{~d} v_{j}\left[\nabla_{\alpha} V\left(\left|r_{\alpha}-r_{j}\right|\right)\right] f_{1}\left(r_{\alpha}, v_{\alpha ;} t\right) \\
& \quad \times\left[f_{1}\left(r_{j}, v_{j} ; t\right)-c \varphi\left(v_{j} ; t\right)\right] \\
& \quad=I_{\mathrm{cor}}^{\prime}\left(r_{\alpha}, v_{\alpha} ; t\right)
\end{aligned}
$$

where 


$$
I_{\text {cor }}^{\prime}\left(r_{\alpha}, v_{\alpha} ; t\right)=\frac{1}{2} e^{2} c m^{-1} \partial_{\alpha} \cdot \int \mathrm{d} r_{j} \mathrm{~d} v_{j}\left[\nabla_{\alpha} V\left(\left|r_{\alpha}-r_{j}\right|\right)\right] G\left(r_{\alpha}-r_{j} ; \boldsymbol{v}_{\alpha}, \boldsymbol{v}_{j} ; t\right)
$$

and

$$
\begin{aligned}
G\left(r_{\alpha}-r_{j}, v_{\alpha}, v_{j} ; t\right)= & \frac{2}{c}\left[g\left(r_{\alpha}-r_{j}\right)-1\right]\left[f_{1}\left(r_{\alpha}, v_{\alpha} ; t\right) f_{1}\left(r_{j}, v_{j} ; t\right)\right. \\
& \left.-c f_{1}\left(r_{\alpha}, v_{\alpha} ; t\right) \varphi\left(v_{j} ; t\right)\right]
\end{aligned}
$$

The function $G\left(r_{\alpha}-r_{j}, v_{\alpha}, v_{j} ; t\right)$ is the correlation function [16] in the approximation (1).

We can write eq. (14) in terms of a $r$-Fourier transform, i.e.

$$
I_{\text {cor }}^{\prime}\left(r_{\alpha}, v_{\alpha} ; t\right)=\frac{\Omega}{4 \pi^{2}} \int \mathrm{d} k \mathrm{e}^{\mathrm{i} k \cdot r_{\alpha}} I_{\text {cor }}^{\prime}\left(k, v_{\alpha} ; t\right),
$$

where

$$
I_{\text {cor }}^{\prime}\left(k, v_{\alpha} ; t\right)=\frac{4 \pi^{2}}{\Omega} e^{2} c m^{-1} \partial_{\alpha} \cdot \int \mathrm{d} k^{\prime} \mathrm{d} v_{j} F_{k-k^{\prime}}, \rho_{k+k^{\prime},-k-k^{\prime}} \delta(k)
$$

and $F_{k-k^{\prime}}$ is the Fourier transform of the function $\nabla_{\alpha} V\left(\left|r_{\alpha}-r_{\beta}\right|\right)$.

Comparing eq. (12) with eq. (14), we obtain that the equation proposed by STLS is derived from GVE if

$$
\rho_{\left[k^{\prime}, k-k^{\prime}\right]} \equiv \rho_{k+k^{\prime},-k-k^{\prime}} \delta(k),
$$

where $\delta(k)$ is the $\delta$-Dirac function.

Eq. (18) shows that in the STLS approximation the true correlation coefficient $\rho_{\left[k^{\prime}, k-k^{\prime}\right]}$, as a function of $k$, is sharply peaked at $k=0$. This fact is in agreement with the general analysis of the Fourier components in DC theory. Indeed, in this analysis, the two-particle correlation functions $G\left(r_{\alpha}, r_{\beta}, w_{\alpha}\right.$, $\left.\boldsymbol{v}_{\beta} ; t\right)$ is witten in terms of the center of mass $(R)$ and relative $(r)$ coordinates by [16]

$$
\begin{aligned}
G\left(\boldsymbol{R}, \boldsymbol{r}, \boldsymbol{v}_{\alpha}, \boldsymbol{v}_{\beta} ; t\right)= & \int \mathrm{d} \boldsymbol{k}_{1} \mathrm{~d} \boldsymbol{k}_{2} \exp \left\{\mathrm{i}\left(k_{1}+\boldsymbol{k}_{2}\right) \cdot \boldsymbol{R}+\mathrm{i}\left(k_{1}-\boldsymbol{k}_{2}\right) \cdot \boldsymbol{r}\right\} \\
& \times \rho_{\left|\boldsymbol{k}_{1}, k_{2}\right|}\left(\boldsymbol{v}_{\alpha}, \boldsymbol{v}_{\beta} ; t\right) .
\end{aligned}
$$

The variation of $G$ with the coordinate $R$ of the center of mass of the couple of particles $(\iota, \beta)$ is slow in molecular scale, $L_{\mathrm{m}}$, if $L_{\mathrm{h}} \gg L_{\mathrm{m}}\left(L_{\mathrm{h}}\right.$ is the hydrodynamic length scale and $L_{\mathrm{m}}$ the largest characteristic molecular length scale). 
Thus, $\rho_{\left[k_{1}, k_{2}\right]}\left(v_{\alpha}, v_{\beta}\right)$ as a function of $k_{1}+k_{2}$ is sharply peaked around $k_{1}+$ $k_{2}=0$, with a width of order $L_{\mathrm{h}}^{-1}$ [16]. In our case, $k_{1}=k^{\prime}$ and $k_{2}=k-k^{\prime}$. Then, we have $k_{1}+k_{2}=k$, i.e. $\rho_{\left[k^{\prime}, k-k^{\prime}\right]}$ must be sharply peaked at $k=0$, as indicated in eq. (18).

On the other hand, $\rho_{\left[k^{\prime}, k-k^{\prime}\right]}$, as function of $k^{\prime}$, has for $k=0$ the behaviour of $\rho_{k^{\prime},-k^{\prime}}$, i.e., $\rho_{\left[k^{\prime}, k-k^{\prime}\right]}$ has for $k=0$ the same physical nature as the Fourier components, which describe correlations in a homogeneous system. It follows from our development, by using DC theory, that the STLS approach corresponds to the study of a non-homogeneous system by considering the correlations as given in a homogeneous system.

\section{Concluding remarks}

We have derived a generalized Vlasov equation (GVE) by using the DC theory developed mainly by Prigogine and Balescu. From GVE we have obtained the condition which the true correlation coefficient $\rho_{\left[k^{\prime}, k-k^{\prime}\right]}$ must satisfy in STLS approximation, i.e.,

$$
\rho_{\left[k^{\prime}, k-k^{\prime}\right]} \equiv \rho_{k+k^{\prime},-k-k^{\prime}} \delta(k) .
$$

This relation shows that in STLS approximation $\rho_{\left[k^{\prime}, k-k^{\prime}\right]}$ is sharply peaked around $k=\boldsymbol{0}$ and that, for $k=\boldsymbol{0}, \rho_{\left[k^{\prime}, k-k^{\prime}\right]}=\rho_{k^{\prime},-k^{\prime}}$. Hence, we can say that STLS approximation describes a non-homogeneous system by considering the correlations as a homogeneous system.

Our development shows also by analyzing the term $I_{\text {cor }}(r, v ; t)$, in eq. (11), that it is possible to use other approximations. For example, we can consider a velocity-depending true correlation coefficient. Such an approximation will be an improvement to the STLS approach.

\section{Acknowledgement}

One of us (AES) was partially supported by CNPq (a Brazilian Government Agency).

\section{References}

[1] T. Ando, Rev. Mod. Phys. 54 (1982) 437.

[2] M.W. Cole, Rev. Mod. Phys. 46 (1974) 451.

[3] V.B. Shikin and Yu.P. Monarkha, Sov. J. Low Temp. 1 (1975) 459 (Fiz. Nizk. Temp. 1 (1975) 957). 
[4] N. Studart and O. Hipólito, Rev. Bras. Fis. 16 (1986) 194.

[5] C.G. Grimes, Suriace Science 73 (1978) 379.

[6] A.L. Fetter, Phys. Rev. B 10 (1974) 3739.

[7] P.M. Platzmann and N. Tzoar, Phys. Rev. B 13 (1976) 3197.

[8] H. Totsuji, J. Phys. Soc. Japan (Lett.) 39 (1975) 253.

[9] H. Totsuji, J. Phys. Soc. Japan 40 (1976) 857.

[10] M. Bauss, J. Stat. Phys. 19 (1978) 163.

[11] L. Sjogren, J. Phys. C; Solid State Phys. (Lett.) 13 (1980) L841.

[12] N. Studart, O. Hipolito, Phys. Rev. A 19 (1979) 1790.

[13] N. Studart, O. Hipolito, Phys. Rev. A 22 (1980) 2860.

[14] K.S. Singwi, M.P. Tosi, R.H. Land and A. Sjolander, Phys. Rev. 176 (1968) 589.

[15] I. Prigogine, Non-equilibrium Statistical Mechanics (Wiley, New York, 1962).

[16] R. Balescu, Statistical Mechanics of Charged Particles (Interscience, New York, 1963).

[17] Y. Soulet and A. Gomes, J. Stat. Phys. 25 (1981) 695.

[18] A.A. Vlasov, Zh. Exp. Teor. Fiz. 8 (1938) 231.

[19] L.E. Reichl, A Modern Course in Statistical Physics (Univ. Texas Press, Austin, 1984). 